\title{
Analytical Modeling of an Oblique Edge Crack in Rolling Contact Fatigue
}

\author{
Francesca Di Puccio \\ Dipartimento di Ingegneria Civile e Industriale, Università di Pisa, Largo Lazzarino 2, 56126 Pisa, Italy \\ Correspondence should be addressed to Francesca Di Puccio; dipuccio@ing.unipi.it
}

Received 13 December 2017; Accepted 17 February 2018; Published 27 March 2018

Academic Editor: Fabrizio Greco

Copyright (C) 2018 Francesca Di Puccio. This is an open access article distributed under the Creative Commons Attribution License, which permits unrestricted use, distribution, and reproduction in any medium, provided the original work is properly cited.

\begin{abstract}
Surface cracks represent a frequent cause of damage and even failure in rolling contacts, observed in gears, cams, rails, and so on. In the literature, different approaches have been applied to describe the crack behaviour by means of Fracture Mechanics parameters, such as the stress intensity factors (SIFs) and the $J$-integral. In this paper, a general procedure for dealing with plane problems is presented, which is based on Linear Elastic Fracture Mechanics hypotheses. It combines the Weight Function Method for evaluating the SIFs in a loading cycle with the Kolosov-Muskhelishvili complex variable approach for estimating the nominal stress field. In this way, a completely analytical procedure can be applied for a general loading condition, assuming that the real geometry can be simplified in a half-plane with an oblique edge crack. As test case, a travelling load has been considered representing a combination of three contributions: Hertzian pressure distribution, traction force due to friction, and pressurization of the crack faces. A comparison with literature results proved that the proposed approach can be an efficient tool for SIFs estimation and crack growth description.
\end{abstract}

\section{Introduction}

It is well known that rolling contact fatigue may be a primary cause of failure in many mechanical applications, such as cams, gears, and railway tracks, as already observed by Tallian in 1969 [1]. Damage processes as pitting, spalling, fretting [2], or even catastrophic failures [3] are frequently attributed to fatigue phenomena. This topic has attracted many researchers since the 1980s, for example, [4-7], but a renewed interest has characterized the last decade, for example, [8-11]. In most of the studies in the literature, the rolling contact between two bodies is simplified as a two-dimensional problem of a half-space with a travelling pressure distribution over its surface. Repeated loading, that is, repeated passages of such pressure distribution, causes initiation of fatigue cracks from defects or inclusions below or directly from the surface. Experimental results show cases of fatigue cracks propagating in the load movement direction with a small angle with respect to the surface $\left(\boldsymbol{\alpha} \approx 20^{\circ}-30^{\circ}\right)$ [5]. However, when only pressure distribution is considered, crack propagation should occur in a compressive field that does not actually explain experimental observations. Thus, other actions have to be taken into account, starting from a "tractive" tangential force along the surface. Bower in [7] pointed out two additional mechanisms influencing crack propagation in wet cases: the lubricant action on the crack faces inducing a "hydraulic pressure mechanism" and the presence of trapped fluid inside the crack. All these conditions complicate the theoretical investigation of the phenomenon.

In [8], a survey of some approaches applied to the twodimensional problem is presented, focused on the estimation of the stress intensity factors (SIFs), which represent the main parameters for describing the crack behaviour in Linear Elastic Fracture Mechanics [12, 13]. Keer and Bryant initially proposed the application of the dislocation method [4] subsequently followed by Bower [7] and more recently by Datsyshyn and Marchenko [8]. Murakami and Nemat-Nasser introduced the so-called "body force method" in 1983 also applied to a 3D case [6]. After Bogdański et al. in 1996 [14], the finite element method has been frequently applied in these problems, as $[11,15]$.

The present paper describes a procedure based on the application of the Weight Function (WF) Method to evaluate the SIFs in rolling contact plane problems. The approach 


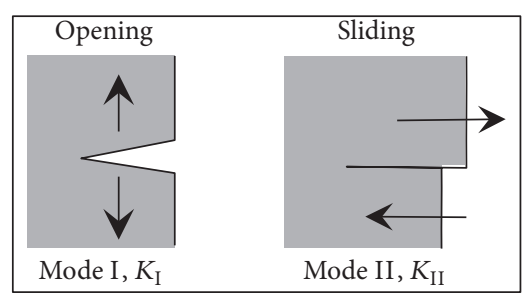

(a)

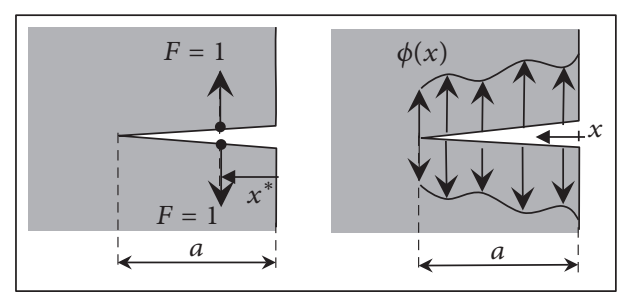

(b)

FIGURE 1: (a) Crack fracture modes in plane; (b) left: couple of unitary forces opening the crack; right: general traction distributions on the crack faces.

was initially applied by the author in 1999 [16] to compare the effects of Hertzian and Elasto-Hydrodynamic pressure distribution on the SIFs in a loading cycle. More recently, the use of WFs has been reproposed by Beghini et al. in $[9,17]$. They combined the WFs with a discretization approach to estimate the nominal stress field while, in this study as in [16], the Kolosov-Muskhelishvili complex variable method is considered.

The aim of this study is to present the theoretical foundations and the implementation features of the proposed approach since it represents a powerful tool for investigating this type of problems and can be easily extended to different loading conditions.

\section{Theoretical Background}

In Linear Elastic Fracture Mechanics, it is assumed that the behaviour of a crack is governed by the elastic stress field at its tip, which is characterized by the stress intensity factors (SIFs), commonly denoted as $K$. This means that the effect of the loading conditions on the crack propagation may be evaluated in terms of $K$.

In a general plane problem, two types of fracture modes can be distinguished: the opening of the crack faces (mode I) and their relative sliding (mode II), shown in Figure 1(a). For completeness, we recall that a third mode is present in $3 \mathrm{D}$ cases. For each mode, a SIF is defined, labelled as $K_{\mathrm{I}}$ or $K_{\mathrm{II}}$, respectively. In a loading cycle, the behaviour of a plane crack depends on the time history of the two SIFs that represents a measure for assessing rolling contact fatigue. Thus, SIF evaluation is a key issue in these problems.

2.1. The Weight Functions Method. The WF Method offers a powerful tool for evaluating the SIFs since it requires a rather simple stress analysis and, obviously, the knowledge of the WFs that for many cases are available in the literature [20, 21].

In order to give a brief overview of the physical meaning of the WFs, let us consider a simple geometry shown in Figure 1(b) (left), representing a panel with a crack of length $a$ loaded by a couple of unitary forces $F$ at a distance $x^{*}$ from the crack mouth. Since this is a symmetrical opening action for the crack, the crack behaviour depends only on $K_{\mathrm{I}}$ that can be estimated as $K_{\mathrm{I}}=h_{\mathrm{I}}\left(x^{*}, a\right)$, where $h_{\mathrm{I}}(x, a)$ is the WF of this specific case. Conversely, $h_{\mathrm{I}}(x, a)$ represents the SIF for the geometry under examination produced by a couple of unitary forces applied in $x$. Due to the linearity of the problem, the superposition principle can be applied to evaluate the SIF for a general distribution $\phi(x)$ of normal actions on the crack faces shown in Figure 1(b) (right); that is,

$$
K_{\mathrm{I}}=\int_{0}^{a} h_{\mathrm{I}}(x, a) \phi(x) d x .
$$

However, the WFs Method can be applied also for a general system of forces, not limited to tractions on the crack faces. The idea is described in Figure 2(a), representing a double edge cracked panel in tension (again mode I). According to the superposition principle, the SIF produced in the cracked panel may be considered as the sum of the one produced in the uncracked panel by the external applied loads (no crack implies $K_{\mathrm{I}}=0$ ) and the one caused by a stress distribution acting on the crack lips. It can be observed that such a stress distribution is opposite to the one acting in the integer panel (nominal stress field), as the crack faces in the cracked panel are free. Combining this result with the above described meaning of the WFs, the SIF for the cracked panel can be calculated as

$$
K_{\mathrm{I}}=\int_{0}^{a} h_{\mathrm{I}}(x, a) \sigma_{n}(x) d x .
$$

In this way, once the WF is known for the geometry under examination, it is sufficient to evaluate the nominal normal stresses $\sigma_{n}(x)$ in the integer body for determining the SIF. The extension to mode II loading is straightforward, $K_{\text {II }}$ being connected, in a symmetrical geometry, to the nominal shear stresses $\tau_{n}(x)$; that is,

$$
K_{\mathrm{II}}=\int_{0}^{a} h_{\mathrm{II}}(x, a) \tau_{n}(x) d x
$$

Two points about the sign of the SIFs are worth being underlined. Positive nominal normal stresses $\sigma_{n}(x)$ in the uncracked panel correspond to tensile stresses and consequently to compressive actions on the crack lips, as in Figure 2(a) (right). These compressive actions produce a positive $K_{\mathrm{I}}$. Thus, in (2), the sign of nominal stresses (plus) is maintained and not reversed. Conversely, compressive nominal normal stresses mean tractive forces on the crack faces that would close the crack causing contact between the lips. Such contact actions guarantee that, in any case, $K_{\mathrm{I}} \geq 0$. Moreover, the sign of $K_{\mathrm{II}}$ is arbitrary as the sign of shear stresses $\tau_{n}$ and they are chosen coherently so that when $\tau_{n}>0$ also $K_{\mathrm{II}}>0$ and vice versa. 


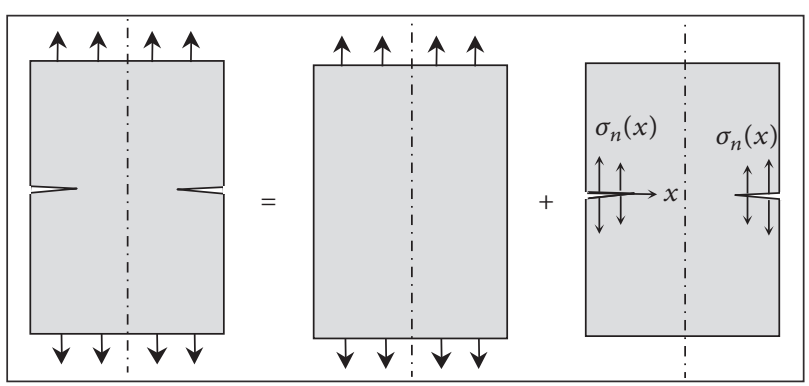

(a)

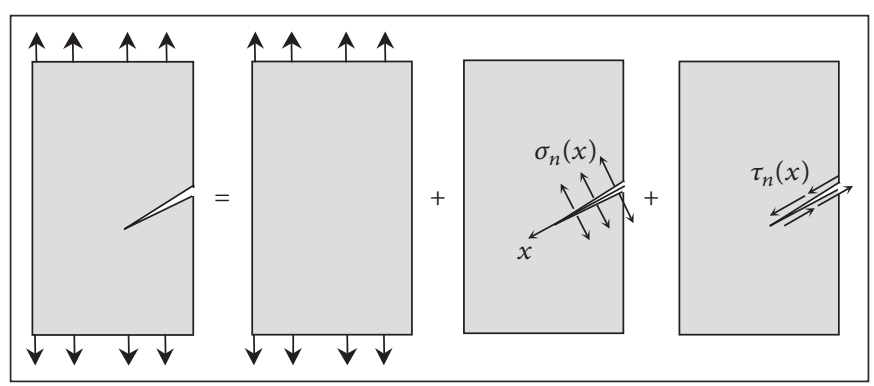

(b)

FIGURE 2: (a) Superposition principle for the SIF in a cracked panel. (b) Panel with an oblique crack in tension.

When the geometry is not symmetrical as in the previous example, for example, when the crack is oblique as in Figure 2(b), a coupled influence of normal and shear stresses on $K_{\mathrm{I}}$ and $K_{\mathrm{II}}$ has to be taken into account and the complete SIF expressions become

$$
\begin{aligned}
K_{\mathrm{I}} & =\int_{0}^{a}\left[h_{\mathrm{I}, \sigma}(x, a) \sigma_{n}(x)+h_{\mathrm{I}, \tau}(x, a) \tau_{n}(x)\right] d x, \\
K_{\mathrm{II}} & =\int_{0}^{a}\left[h_{\mathrm{II}, \sigma}(x, a) \sigma_{n}(x)+h_{\mathrm{II}, \tau}(x, a) \tau_{n}(x)\right] d x .
\end{aligned}
$$

It must be underlined that, for these more complex cases, the WFs have been reported rather recently in the literature and only for few geometries, as a cracked half-plane $[18,19]$.

2.2. Stress Field in a Half-Plane. Let us consider a homogenous, isotropic linear elastic half-plane, lying in the part $y \leq 0$ of a Cartesian $x-y$ frame. Neglecting volume force distributions, the stress field can be described using the socalled Airy's function $A(x, y)$ [22] as

$$
\begin{gathered}
\sigma_{x x}=\frac{\partial^{2} A(x, y)}{\partial y^{2}}, \\
\sigma_{y y}=\frac{\partial^{2} A(x, y)}{\partial x^{2}}, \\
\sigma_{x y}=\frac{\partial^{2} A(x, y)}{\partial x \partial y}
\end{gathered}
$$

holding in both plane stress and plane strain conditions. In these cases, the first invariant of the stress tensor must satisfy the following relationship:

$$
\begin{aligned}
\left(\frac{\partial^{2}}{\partial x^{2}}+\frac{\partial^{2}}{\partial y^{2}}\right)\left(\sigma_{x x}+\sigma_{y y}\right) & =0 \\
\text { or } \Delta\left(\sigma_{x x}+\sigma_{y y}\right) & =0
\end{aligned}
$$

so that Airy's function must be of class $\mathrm{C}^{4}$ and biharmonic; that is,

$$
\Delta \Delta A(x, y)=0
$$

Introducing the complex variable $z=x+i y$, with $\bar{z}$ its complex conjugate, $A(z)$ can be written starting from the general expression of a biharmonic function as

$$
\begin{aligned}
\Delta \Delta A(z) & =0 \longrightarrow \\
2 A(z) & =\operatorname{Re}[\bar{z} \varphi(z)+\chi(z)],
\end{aligned}
$$

where $\varphi(z)$ and $\chi(z)$ are holomorphic functions. Accordingly, the stress components are related through the following equations:

$$
\begin{aligned}
\sigma_{x x}+\sigma_{y y} & =4 \operatorname{Re}\left[\varphi^{\prime}(z)\right]=4 \operatorname{Re}[\Phi(z)], \\
\sigma_{y y}-\sigma_{x x}+2 i \sigma_{x y} & =2\left[\bar{z} \varphi^{\prime \prime}(z)+\chi^{\prime}(z)\right] \\
& =2\left[\bar{z} \Phi^{\prime}(z)+\Psi(z)\right],
\end{aligned}
$$

where $\Phi(z)=\varphi^{\prime}(z)$ and $\Psi(z)=\chi^{\prime}(z)$, known as Muskhelishvili's potential functions, are conveniently introduced [23].

Given the normal $N$ and shear $T$ load distributions on the boundary (i.e., along the $x$-axis), the two potentials can be calculated as

$$
\begin{aligned}
& \Phi(z)=-\frac{1}{2 \pi i} \int_{-\infty}^{\infty} \frac{N(l)-i T(l)}{l-z} d l, \\
& \Psi(z)=-\frac{1}{2 \pi i} \int_{-\infty}^{\infty} \frac{N(l)+i T(l)}{l-z} d l-\Phi(z)-z \Phi^{\prime}(z) .
\end{aligned}
$$

Both $N(l)$ and $T(l)$ have to be continuous and tend to zero (at least as $1 / l)$ when $|l| \rightarrow \infty$. When only normal actions $N$ are applied, the stress tensor components are

$$
\begin{aligned}
& \left\{\begin{array}{l}
\sigma_{y y}=2 \operatorname{Re}[\Phi(z)]+2 y \operatorname{Im}\left[\Phi^{\prime}(z)\right] \\
\sigma_{x x}=2 \operatorname{Re}[\Phi(z)]-2 y \operatorname{Im}\left[\Phi^{\prime}(z)\right] \\
\sigma_{x y}=-2 y \operatorname{Re}\left[\Phi^{\prime}(z)\right]
\end{array}\right. \\
& \stackrel{\text { at the boundary } y=0}{\longrightarrow}\left\{\begin{array}{l}
\sigma_{x x}=\sigma_{y y}=2 \operatorname{Re}[\Phi(x+i 0)] \\
\sigma_{x y}=0 .
\end{array}\right.
\end{aligned}
$$




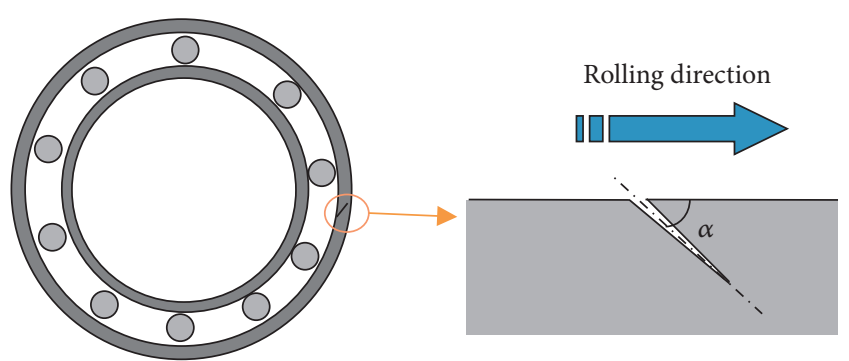

(a)

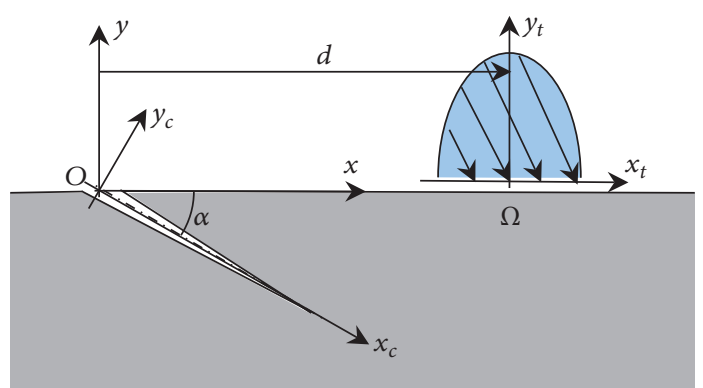

(b)

FIGURE 3: (a) Scheme of the geometry: from the real problem to a cracked half-plane. (b) Reference frames and other symbols.

On the other hand, for only $T$ we obtain

$$
\begin{aligned}
& \left\{\begin{array}{l}
\sigma_{y y}=\operatorname{Re}\left[(\bar{z}-z) \Phi^{\prime}(z)\right] \\
\sigma_{x x}=\operatorname{Re}\left[4 \Phi(z)-(\bar{z}-z) \Phi^{\prime}(z)\right] \\
\sigma_{x y}=\operatorname{Im}\left[(\bar{z}-z) \Phi^{\prime}(z)-2 \Phi(z)\right]
\end{array}\right. \\
& \stackrel{\begin{array}{l}
\sigma_{y y}=0 \\
\sigma_{x x}=4 \operatorname{Re}[\Phi(x+i 0)] \\
\sigma_{x y}=-2 \operatorname{Im}[\Phi(x+i 0)]
\end{array}}{ }
\end{aligned}
$$

\section{Materials and Methods}

3.1. Geometrical Assumptions. Rolling contact fatigue affects many mechanical components, from gears to roller bearings. Each case is characterized by its own geometry and working conditions. However, with some simplifying assumptions, the presented approach can be applied to most of them.

As far as the geometry is concerned, we will assume that the crack length $a$ is much smaller than the main dimension of the problem (e.g., radius of curvature $r \gg a$ ) so that the crack region can be described as a half-plane with a surface crack, as shown in Figure 3(a). A reference frame $S$ on the half-plane is assumed, with origin at the crack mouth (Figure 3(b)), $x$-axis along the boundary, and the body extending over $y \leq 0$, as for (10)-(12). A surface crack usually initiates from the free surface and grows through mode I and II mechanisms at an angle $\alpha$ with respect to the surface. Typical values of $\alpha$ are in the range $20^{\circ}-30^{\circ}$ with the tip of the crack shifted towards the rolling direction with respect to its mouth.

3.2. Weight Functions. For an oblique edge crack in a halfplane, two sources for the WFs can be found: $[18,19]$. In the first study, a solution is provided for $20^{\circ} \leq \alpha \leq 90^{\circ}$ through 80 coefficients of power series expansions, while in the second case 8 coefficients are given, but only for some discrete values of $\alpha\left(15^{\circ}, 30^{\circ}, 45^{\circ}, 60^{\circ}\right.$, and $\left.90^{\circ}\right)$ (see Appendix for complete details). Such functions are compared in Figure 4 for two angles of interest, that is, $\alpha=15^{\circ}$ and $\alpha=30^{\circ}$, for a unitary crack length. It can be observed that the four functions are very similar apart from the mixed term $h_{\mathrm{I}, \tau}(x, a)$ in the range
$0 \leq x / a \leq 0.6$. Actually, they are also proposed assuming a different positive direction for the tangential actions $\tau_{n}$ (and thus for $K_{\mathrm{II}}$ ), as detailed in Appendix, so care should be taken when choosing the WFs.

3.3. Loading Conditions. In this simplified geometry, fatigue actions are described as travelling loads that move along the $x$-axis. In rolling contact fatigue, travelling loads are usually distinguished in

(a) normal loads ( $N$-type): contact pressures, typically approximated with Hertzian contact formulas,

(b) tractive loads ( $T$-type): tangential actions, representing frictional forces, generally expressed as $f|N|$, with $f$ coefficient of friction. A sign is attributed to $f$ to simulate driving (i.e., in the motion direction, $f>0$ ) or driven (i.e., opposite to the motion direction, $f<$ $0)$ conditions.

We will assume that these loads simply translate along the $x$-axis maintaining their characteristics. Thus, a second reference frame $S_{t}\left\{\Omega, x_{t}, y_{t}\right\}$ is introduced where they can be easily defined and remain constant. Accordingly, the normal load due to Hertzian pressure can be expressed as

$$
N\left(x_{t}\right)=-p_{0} \sqrt{1-\left(\frac{x_{t}}{a_{H}}\right)^{2}} \text { for }-a_{H} \leq x_{t} \leq a_{H},
$$

where $p_{0}$ is the maximum pressure and $a_{H}$ the contact half-width. The minus sign indicates a compressive action. Therefore, the tangential load is

$$
T\left(x_{t}\right)=f p_{0} \sqrt{1-\left(\frac{x_{t}}{a_{H}}\right)^{2}} .
$$

In dry conditions, these are the external loads. Additionally, contact actions $\sigma_{c}$ between the crack lips can arise to avoid their overlapping as a consequence of compressive forces. Actually, the crack can be totally or partially closed, depending also on the crack size and on the load position (in particular on the ratio $\left.a / a_{H}\right)$. A correct formulation of the problem can be found, for example, in $[8,11]$, and introduces important complexities to the solution passing through the estimation of the displacement field (not only at the crack tip). 

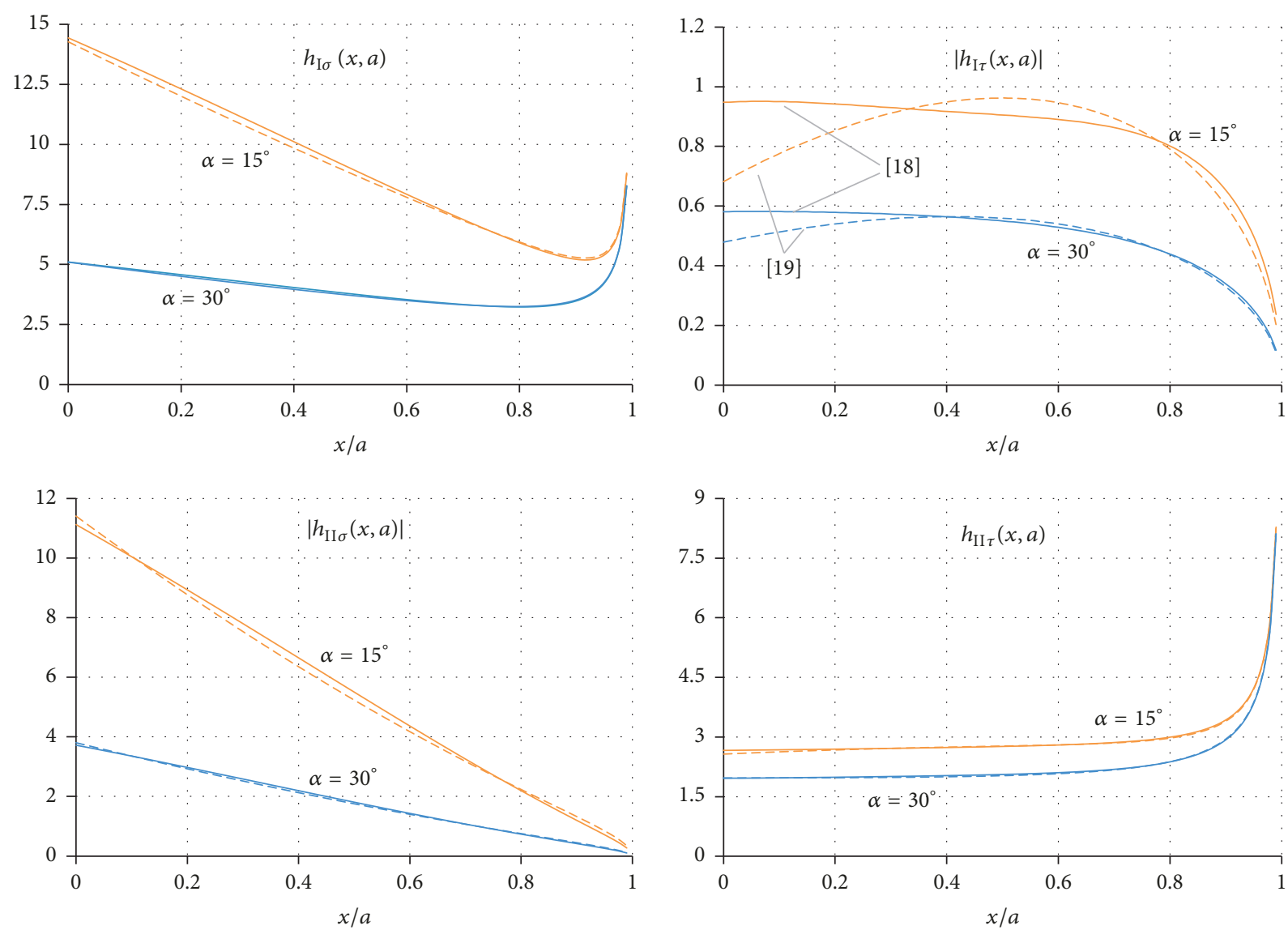

FIGURE 4: Comparison of the WFs from [18, 19] for an oblique edge crack in a half-plane for $\alpha=15^{\circ}$ and $\alpha=30^{\circ}, a=1$. (solid line [18], dashed line [19]).

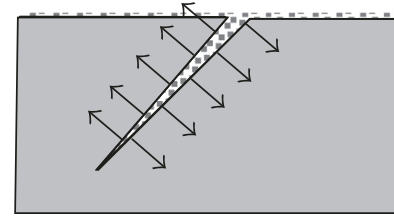

Hydraulic pressure

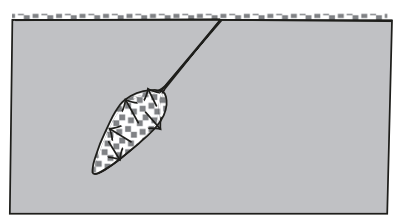

Trapped fluid
FIGURE 5: Actions on the crack faces due to the presence of fluid.

Thus, most frequently general cases are studied with finite element analyses, as in [11]. In this paper, a simplification proposed in $[9,16]$ is adopted, which can be useful for dealing with short cracks, where the crack is mainly open or totally closed. The simplification assumes a proportionality between those contact actions $\sigma_{c}$ on the crack lips and the nominal normal tractions $\sigma_{n}$. The scale factor between $\sigma_{n}$ and $\sigma_{c}$ is calculated imposing $K_{\mathrm{I}}=0$, as described in Section 3.4 (see (20)-(21)). Contact actions can imply further frictional tangential tractions between the crack lips that can be in stick or slip condition $[7,24]$.

In the so-called wet conditions, hydraulic loads due to the presence of fluid (lubricant) within the crack must also be considered. They can affect crack propagation in a double way, as shown in Figure 5: direct "hydraulic pressure mechanism" and entrapment of the fluid inside the crack as stated by Bower in [7].

The description of the first effect requires an assumption on the value of fluid pressure within the crack. Generally, it is considered uniform and equal to the pressure at the crack mouth (as in [7]), or varying linearly from the mouth to the tip where it becomes zero (as in [25]). Both cases can be easily considered with the WFs.

On the other side, fluid entrapment is quite difficult to be evaluated even if it represents a very critical condition for defects propagation. It takes into account the fact that as the contact passes over the crack mouth, a local closure of the faces may be observed in rather long cracks, that is, with $a_{H}<a$. In this case, for some time, the lubricant may remain trapped inside the crack though the lips are closed at the mouth. A volume conservation for the lubricant is usually considered in order to evaluate the actions at the crack tip. As already stated, dealing with contact between the crack lips results in important complications, particularly when the conservation of volume must be guaranteed. However, the condition of fluid entrapment can be considered less dangerous than the crack pressurization as it is assumed that pressure in the trapped fluid is lower than the external one.

3.4. Model Implementation. The model of the oblique surface crack has been written in a completely symbolic form in 
Mathcad. Equations (10)-(14) have been used to estimate the nominal stress field that, for a given load, will be written as a function of $x_{t}$ and $y_{t}$. Then, the stress tensor components in $S$ are simply obtained replacing $x_{t} \rightarrow x-d$ and $y_{t}=y$, obtaining the symbolic functions

$$
\begin{aligned}
& \sigma_{y y}=\sigma_{y y}(x, y, d), \\
& \sigma_{x x}=\sigma_{x x}(x, y, d), \\
& \sigma_{x y}=\sigma_{x y}(x, y, d) .
\end{aligned}
$$

Then, since the nominal normal and shear stresses are required along the crack faces for applying (4), the stress tensor $\mathbf{T}$ must be expressed in a rotated reference frame $S_{c}\left\{O, x_{c}, y_{c}\right\}$ with $O$, at the crack mouth and $x_{c}$ along the crack (Figure 3(b)). That can be done as

$$
[\mathbf{T}]_{S c}=\mathbf{R}[\mathbf{T}]_{S} \mathbf{R}^{T}
$$

where, for positive $\alpha$,

$$
\mathbf{R}=\left[\begin{array}{cc}
\cos \alpha & -\sin \alpha \\
\sin \alpha & \cos \alpha
\end{array}\right] .
$$

Then, the nominal normal and shear stresses on the crack lip $\left(\sigma_{n}\right.$ and $\tau_{n}$ ) having normal $y_{c}$ can be written as a function of $x_{c}$ and $d$, replacing $x=x_{c} \cos \alpha y=-x_{c} \sin \alpha$, obtaining

$$
\begin{aligned}
& \sigma_{n}(x, y, d) \\
& =\sigma_{x x}(x, y, d) \sin ^{2} \alpha+\sigma_{y y}(x, y, d) \cos ^{2} \alpha \\
& \quad+2 \sigma_{x y}(x, y, d) \cos \alpha \sin \alpha \\
& \sigma_{n}\left(x_{c}, d\right) \longleftrightarrow \sigma_{n}\left(x_{c} \cos \alpha,-x_{c} \sin \alpha, d\right) \\
& \tau_{n}(x, y, d) \\
& =\left[\sigma_{x x}(x, y, d)-\sigma_{y y}(x, y, d)\right] \cos \alpha \sin \alpha \\
& \quad+\sigma_{x y}(x, y, d)\left(\cos 2 \alpha-\sin ^{2} \alpha\right) \\
& \tau_{n}\left(x_{c}, d\right) \longleftrightarrow \tau_{n}\left(x_{c} \cos \alpha,-x_{c} \sin \alpha, d\right) .
\end{aligned}
$$

It can be noticed that positive values of $\sigma_{n}$ and $\tau_{n}$ mean stresses in agreement with the direction of $y_{c}$ and $x_{c}$, respectively. At this point, the SIFs can be calculated by means of (4), changing the sign of $\tau_{n}$ if the WFs from [19] are chosen, that is, as anticipated in Section 3.2 and detailed in the Appendix.

In order to reduce the computational time, it is convenient to select some discrete values of $d$, for example, for $-3 a_{H} \leq d \leq 3 a_{H}$, and of $x_{c}$, for $0 \leq x_{c}<a$. Consequently, the integrands in (4) can be numerically estimated, fitted with a spline in $x_{c}$ and integrated for every value of $d$.

At this point, a check of the obtained SIFs has to be done since, as already stated, $K_{\mathrm{I}}$ must be nonnegative; that is,

$$
\begin{array}{r}
K_{\mathrm{I}}(d)=\int_{0}^{a}\left[h_{\mathrm{I}, \sigma}\left(x_{c}, a\right) \sigma_{n}\left(x_{c}, d\right)\right. \\
\left.+h_{\mathrm{I}, \tau}\left(x_{c}, a\right) \tau_{n}\left(x_{c}, d\right)\right] d x_{c} \geq 0 .
\end{array}
$$

When (19) is not satisfied, it means that the crack is closed and contact actions $\sigma_{c}$ on the crack lips must be introduced. According to the simplification introduced in the previous section, such contact actions are estimated as

$$
\sigma_{c}\left(x_{c}, d\right)=\lambda(d) \sigma_{n}\left(x_{c}, d\right),
$$

where $\lambda(d)$ is calculated imposing $K_{\mathrm{I}}=0$; that is,

$$
\begin{aligned}
& \int_{0}^{a}\left[h_{\mathrm{I}, \sigma}\left(x_{c}, a\right) \sigma_{n}\left(x_{c}, d\right)+h_{\mathrm{I}, \tau}\left(x_{c}, a\right) \tau_{n}\left(x_{c}, d\right)\right] d x_{c} \\
& +\lambda \int_{0}^{a}\left[h_{\mathrm{I}, \sigma}\left(x_{c}, a\right) \sigma_{n}\left(x_{c}, d\right)\right] d x_{c}=0 \longrightarrow \\
& \lambda=-\frac{\int_{0}^{a}\left[h_{\mathrm{I}, \sigma}\left(x_{c}, a\right) \sigma_{n}\left(x_{c}, d\right)+h_{\mathrm{I}, \tau}\left(x_{c}, a\right) \tau_{n}\left(x_{c}, d\right)\right] d x_{c}}{\int_{0}^{a}\left[h_{\mathrm{I}, \sigma}\left(x_{c}, a\right) \sigma_{n}\left(x_{c}, d\right)\right] d x_{c}} .
\end{aligned}
$$

Therefore, also $K_{\mathrm{II}}$ must be recalculated as

$$
\begin{aligned}
& K_{\mathrm{II}}(d)=\int_{0}^{a}\left[(1+\lambda) h_{\mathrm{II}, \sigma}\left(x_{c}, a\right) \sigma_{n}\left(x_{c}, d\right)\right. \\
& \left.\quad+h_{\mathrm{II}, \tau}\left(x_{c}, a\right) \tau_{n}\left(x_{c}, d\right)\right] d x .
\end{aligned}
$$

Due to these contact actions, also frictional loads on the crack faces can arise, whose contribution to the SIFs is frequently negligible and thus not considered in this study.

For wet conditions, fluid pressure on the crack faces $p\left(x_{c}, d\right)$ must be taken into account adding a pressurization contribute to the SIFs $K^{p}$; that is,

$$
\begin{aligned}
& K_{\mathrm{I}}^{p}(d)=\int_{0}^{a}\left[h_{\mathrm{I}, \sigma}\left(x_{c}, a\right) p\left(x_{c}, d\right)\right] d x_{c}, \\
& K_{\mathrm{II}}^{p}(d)=\int_{0}^{a}\left[h_{\mathrm{II}, \sigma}\left(x_{c}, a\right) p\left(x_{c}, d\right)\right] d x_{c} .
\end{aligned}
$$

As already mentioned, $p\left(x_{c}, d\right)$ may be constant $o$ linearly decreasing to zero at the crack tip; that is,

$$
\begin{aligned}
p\left(x_{c}, d\right) & =p_{m}(d) \\
\text { or } p\left(x_{c}, d\right) & =p_{m}(d)\left(1-\frac{x_{c}}{a}\right),
\end{aligned}
$$

where $p_{m}$ is the pressure at the crack mouth, from (13)

$$
p_{m}(d)= \begin{cases}0 & \text { if } d \leq-a_{H} \text { or } d \geq a_{H} \\ p_{0} \sqrt{1-\left(\frac{d}{a_{H}}\right)^{2}} & \text { otherwise. }\end{cases}
$$

Thus, $K^{p}$ can be easily calculated as

$$
\begin{aligned}
K_{M}^{p}(d) & =p_{m}(d) \int_{0}^{a} h_{M, \sigma}\left(x_{c}, a\right) d x_{c} \\
\text { or } K_{M}^{p}(d) & =p_{m}(d) \int_{0}^{a} h_{M, \sigma}\left(x_{c}, a\right)\left(1-\frac{x_{c}}{a}\right) d x_{c}
\end{aligned}
$$

with $M=$ I or II, where the integrals can be estimated in closed form, producing a scalar quantity depending on $a$ and $\alpha$ (see Appendix). 


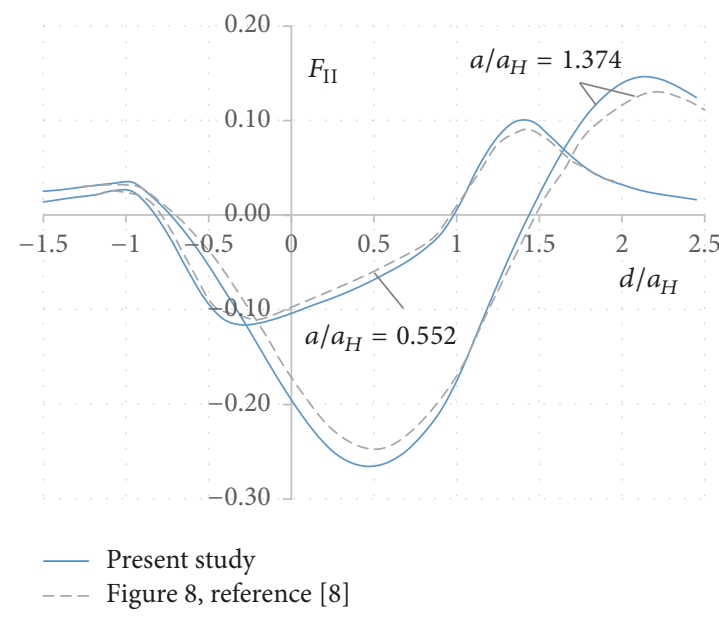

(a)

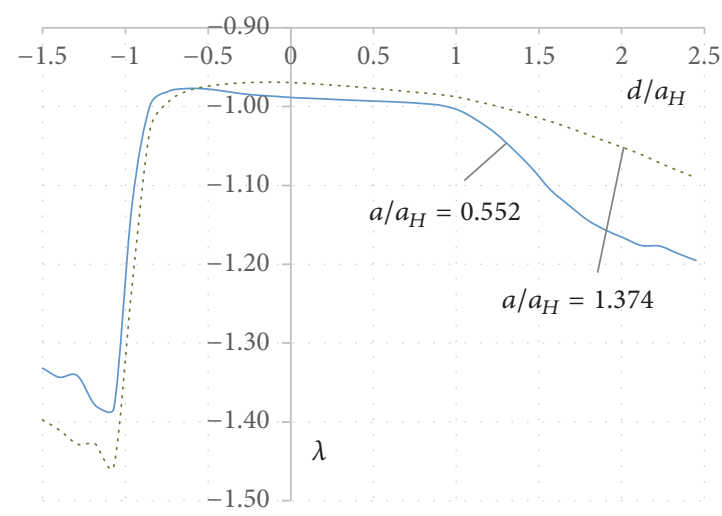

(b)

Figure 6: (a) Dimensionless SIF for mode II, for two crack lengths. Comparison with data of Figure 8 in [8]. (b) Proportionality coefficient for estimating lip contact actions as in (21).

3.5. Numerical Example. In order to validate the proposed model, some comparisons with results from the literature are discussed in Results. Unless stated otherwise, the following numerical data taken from a case used by Datsyshyn and Marchenko [8] has been considered: $\alpha=25^{\circ}, p_{0}=840 \mathrm{MPa}$, $a_{H}=0.22 \mathrm{~mm}$, and $a / a_{H}=0.5$. For this value of $\alpha$ only the WF in [18] can be used.

\section{Results}

Results are usually presented in scaled form, with the dimensionless SIFs written as

$$
F_{\mathrm{I} / \mathrm{II}}=K_{\mathrm{I} / \mathrm{II}} \frac{1}{p_{0} \sqrt{\pi a_{H}}}
$$

plotted versus a dimensionless distance $d / a_{H}$. However, other scaling factors are used in the literature; thus comparisons can require some adaptation.

4.1. Short and Long Crack under Hertzian Contact. At first, a simple case with only Hertzian contact along the boundary has been investigated, taken from [8]. No tractive force $T$ and no friction between the crack lips are considered but the model is applied to compare short and long cracks, that is, $a / a_{H}$ equal to 0.552 and 1.374 , respectively.

Results are shown in Figure 6. Only the dimensionless $F_{\text {II }}$ is reported in Figure 6(a) as $F_{\text {I }}$ is zero. For both crack lengths, the obtained results are in rather good agreement with the literature. Higher errors are observed in peak values, overestimated by the model for about $8 \%$ for the long crack. In Figure 6(b), the values of $\lambda$ estimated according to (21) are depicted. It can be observed that, in the central phase of the contact, that is, when the load passes over the crack mouth $\left(-1<d / a_{H}<1\right), \lambda$ is nearly -1 . This means that $F_{\text {II }}$ is "produced" almost completely by nominal tangential actions, as supposed in [16].

From a computational point of view, each curve is obtained by estimating 25 values in the predefined range $-1.5<d / a_{H}<2.5$ requiring about 15 minutes for all the curves.

4.2. Tractive Loads. The role of motion direction has already been discussed in the literature also in relation to the presence a tractive load $T$ due to nonnegligible friction effect. Results obtained with the proposed approach are compared to those obtained by finite element simulations described in [11].

In Figure $7(\mathrm{a})$, a plot of $F_{\mathrm{II}}$ is shown where a tractive force $T$ is added to the normal one $N$ considering positive and negative values of the friction coefficient. In order to simplify the comparison, that is, to reproduce the given figure, the sign of $F_{\mathrm{II}}$ is modified; thus with respect to Figure 6 curves are turned upside-down. It can be observed that the model can reproduce satisfactorily the trend of the literature.

Figure 7(b) shows the time history of $K_{\mathrm{I}}$ as the load passes over the crack, with different values of the friction coefficient. Tangential actions cause positive values of the first SIF when the load is approaching the crack mouth for driven contact or when the load is leaving for braking contacts, in agreement with the literature (Figures 7(c) and 7(d)).

4.3. Hydraulic Pressure. The effects of fluid pressure on the crack faces have been added, as described in (24)-(26) and (A.3) in Appendix. A constant distribution has been considered, in order to compare the present study with others in the literature.

Results are shown in Figure 8, where the obtained dimensionless SIFs are matched with curves from [11], obtained by FE analyses which in turn are almost overlapped to those by Bower [7] and Beghini and Santus [9]. The effect is obviously limited to the range $-1<d / a_{H}<1$ when the Hertzian pressure at the crack mouth is not zero. As expected, the pressurization of the crack increases markedly $F_{\mathrm{I}}$ (see Figure 6 for comparison with curve for $f=-0.05$ ) and is considered one of the main causes of crack propagation. 


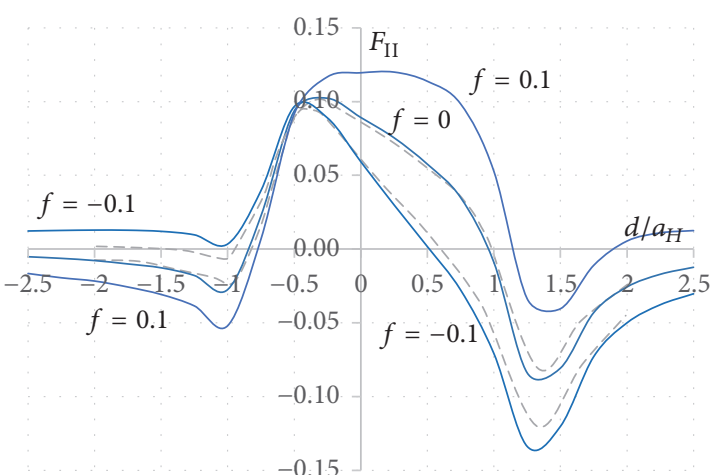

$-0.15$

- Present study

Figure 1A, reference [11]

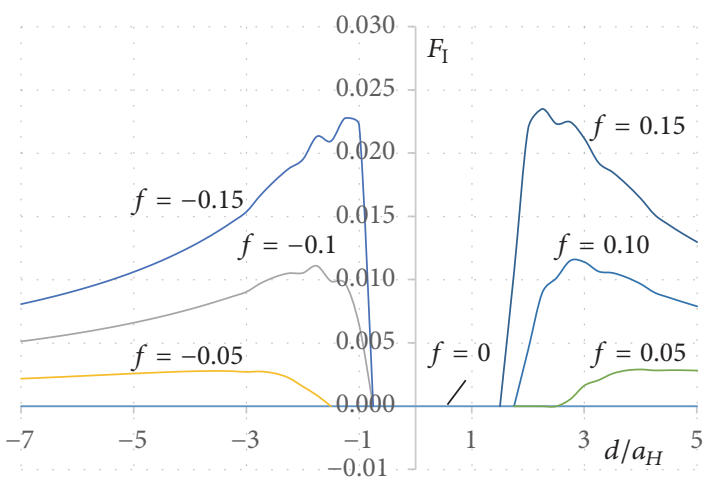

(a)

(b)

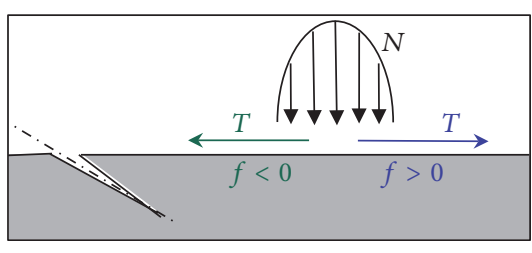

(c)

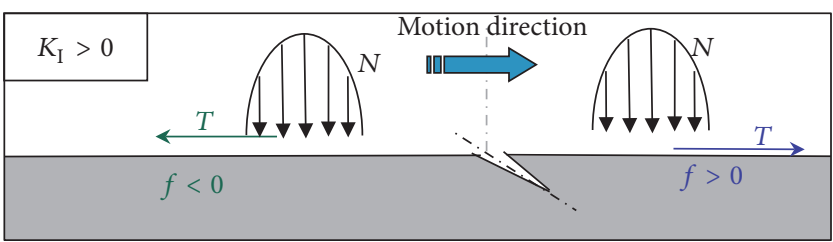

(d)

FIgURE 7: Dimensionless SIFs for mode II (a) and mode I (b), for different values of the friction coefficient. (c) Tangential load direction with $f$. (d) Conditions for positive $K_{\mathrm{I}}$ (numerical case: $a / a_{H}=0.5 ; \alpha=25^{\circ}$ ).

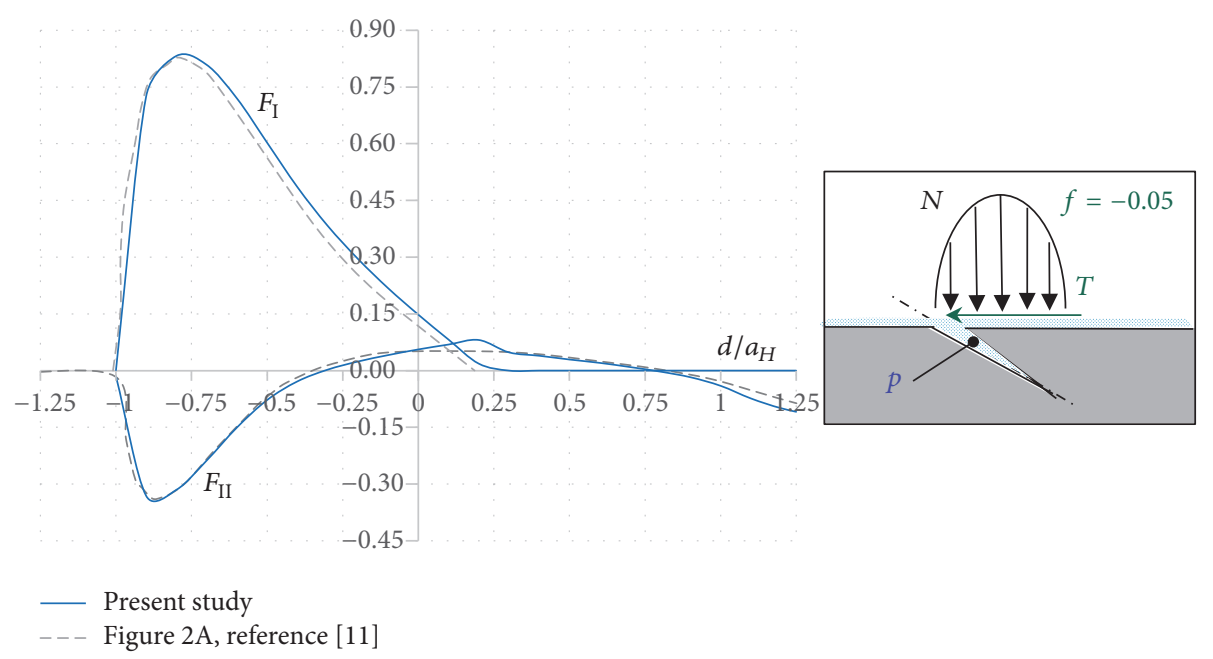

FIGURE 8: Dimensionless SIFs for pressurized crack (numerical case: $a / a_{H}=0.5 ; \alpha=25^{\circ}, f=-0.05$, constant fluid pressure on the crack faces).

4.4. Separate Contributions to the SIFs. As final result, it is interesting to observe an important advantage of this model that enables splitting the single contributions of external load to the SIFs. Figure 8 shows the separated curves of $F_{\mathrm{I}}$ and $F_{\mathrm{II}}$ due to the travelling loads $N$ and $T$ and to the fluid pressure, whose sum gives plots in Figure 9. For $N$ and $T$, the separate contribution of nominal normal $(\sigma)$ and shear stresses $(\tau)$ is also detailed.

The main contribution is produced by the normal stresses due to Hertzian pressure $\left(\sigma_{N}\right)$ and the fluid pressurization, as expected. The two curves have similar magnitude, opposite sign, and a small shift that causes their sum to be positive and negative for $F_{\mathrm{I}}$ and $F_{\mathrm{II}}$, respectively. Other contributions are one order smaller. It is possible to appreciate the positive contribution to $F_{\mathrm{I}}$ of both stress components due to $T$ ( $\sigma_{T}$ and $\tau_{T}$ ) being $f=-0.05$.

This "split" analysis can be useful to achieve fast indications for other cases, such as for increased friction $f=-0.1$, since the superposition principle allows simply doubling the values of the curves due to $\sigma_{T}$ and $\tau_{T}$. 

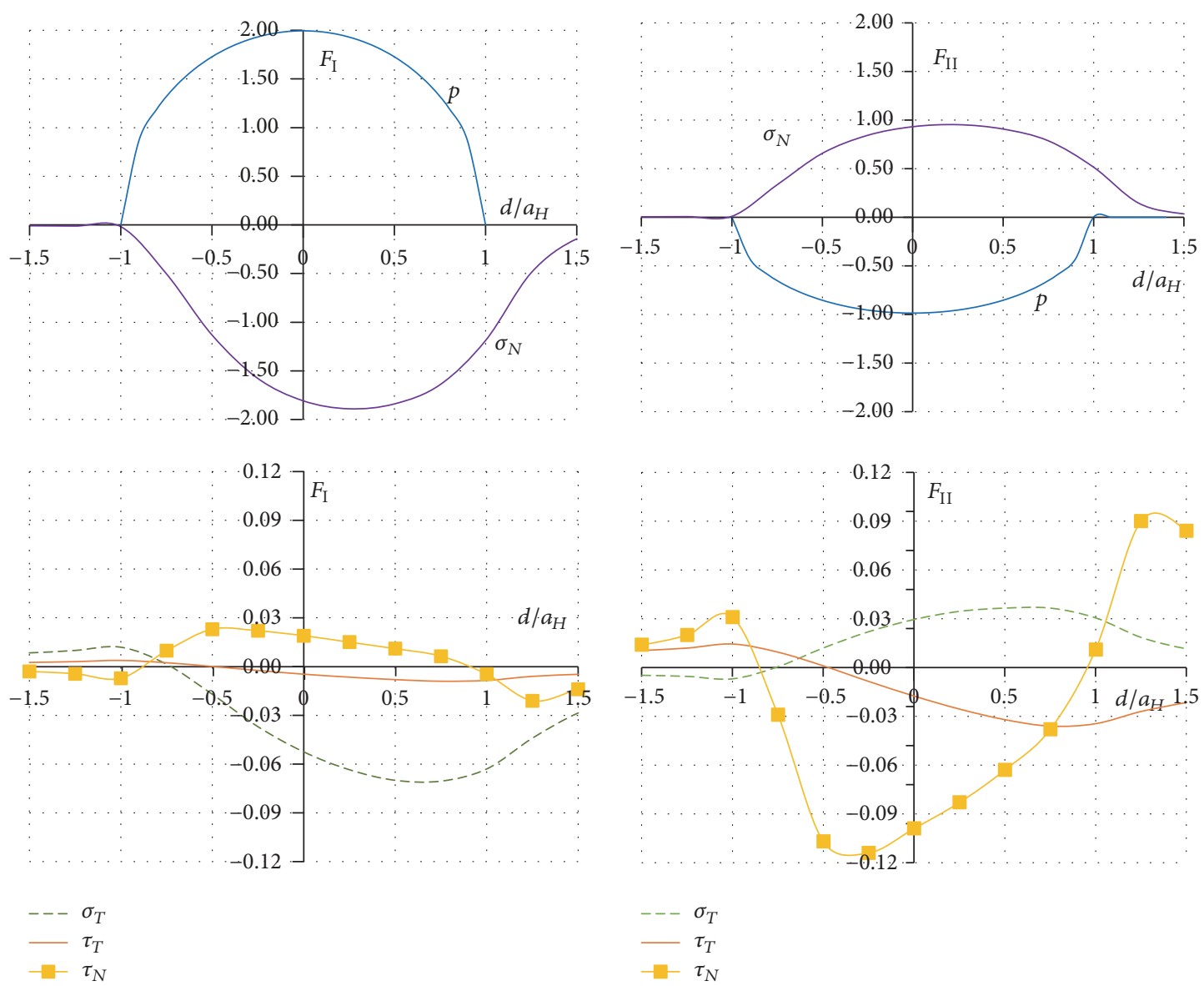

(a)

(b)

FIGURE 9: (a)-(b) Dimensionless SIFs produced by nominal normal $(\sigma)$ and shear stresses $(\tau)$, produced by external loads $N$ and $T$ and by pressure on the crack lips $(p)$ (numerical case: $a / a_{H}=0.5 ; \alpha=25^{\circ}, f=-0.05$, constant fluid pressure on the crack faces).

\section{Conclusions}

An analytical model of a surface crack in a half-plane has been described in detail as well as results of its application compared to other studies in the literature. The model is based on the application of Linear Elastic Fracture Mechanics, in particular taking advantage of the Weight Function Method for evaluating the stress intensity factors which characterize the behaviour and propagation of the crack. The theoretical foundations are also reported in the background.

The model is rather simple to be implemented and its results are in satisfactory agreement with the literature. It can be conveniently applied to investigate many problems of rolling contact fatigue and hopefully it could help to clarify some aspects of this complex phenomenon that are still debated.

\section{Appendix}

\section{Weight Function for an Oblique Crack in a Half-Plane}

The general expressions of the WFs in $[18,19]$ are similar and can be written as functions of the coordinate $x$ along the crack (with origin at the crack mouth), of the crack length $a$, and of the angle $\alpha$ :

$$
\begin{aligned}
& h_{M, \mu}(x, a, \alpha)=\sqrt{\frac{2}{\pi a}}\left[c_{0}^{M, \mu}\left(1-\frac{x}{a}\right)^{-0.5}\right. \\
& \left.+\sum_{i=1}^{4} c_{i}^{M, \mu}(\alpha)\left(1-\frac{x}{a}\right)^{i-0.5}\right],
\end{aligned}
$$

where

$$
c_{0}^{M, \mu}= \begin{cases}1 & \text { for } M, \mu=\mathrm{I}, \sigma \text { or II, } \tau \\ 0 & \text { for } M, \mu=\mathrm{II}, \sigma \text { or I, } \tau\end{cases}
$$

in both [18, 19], while $c_{i}^{M, \mu}=0$ for $i>2$ in [19].

As already stated, the coefficients $c_{i}^{M, \mu}$ are provided as functions of the angle $\theta=\pi / 2-\alpha$ in [18] and for some discrete values of it in [19]. They are plotted in Figure 10(a). As already remarked, the two sources assume different positive directions for the nominal shear stresses, which consequently results also in a different sign of $K_{\mathrm{II}}$. This should be kept in mind when applying the proposed procedure. In Figure 10(b), 

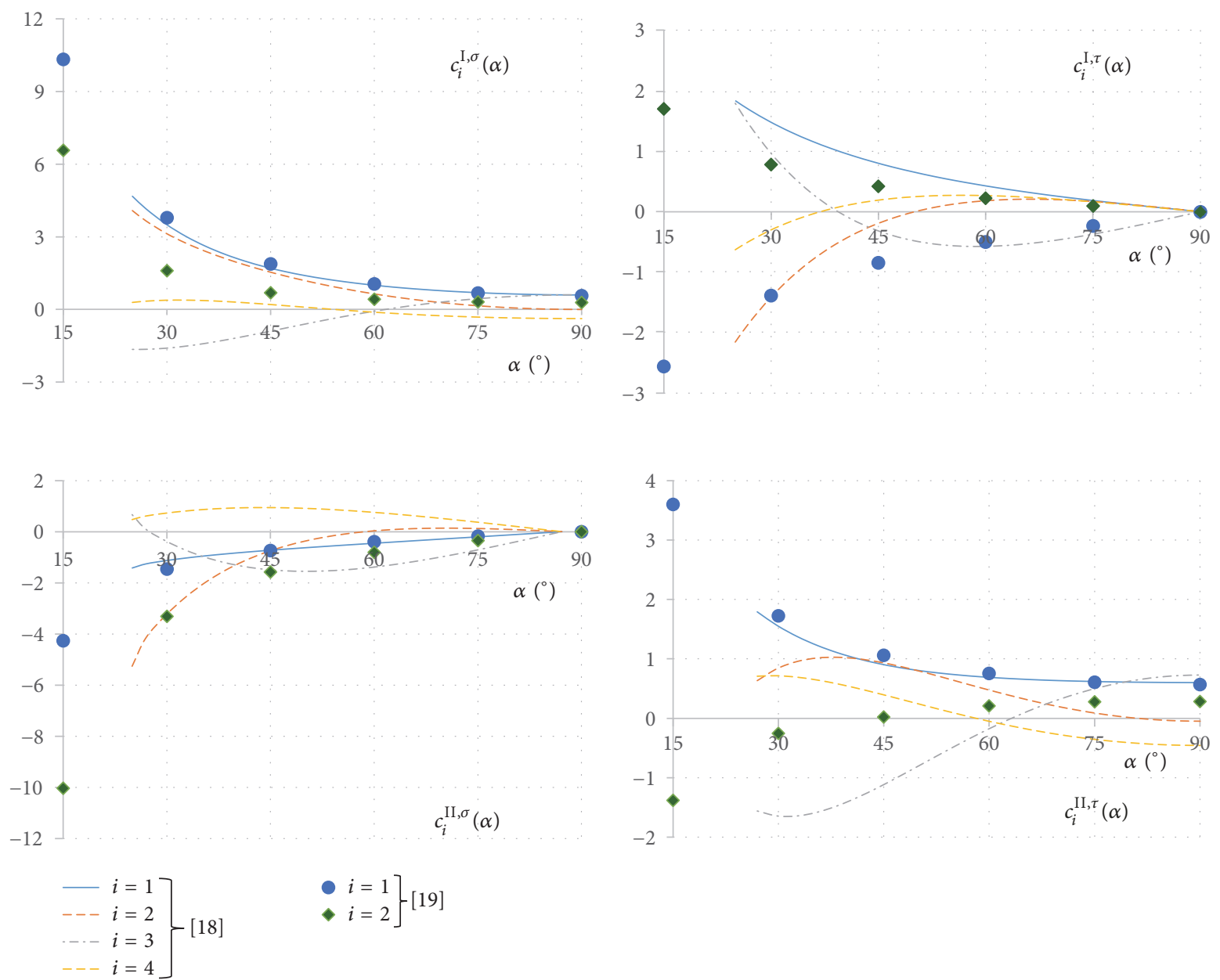

(a)
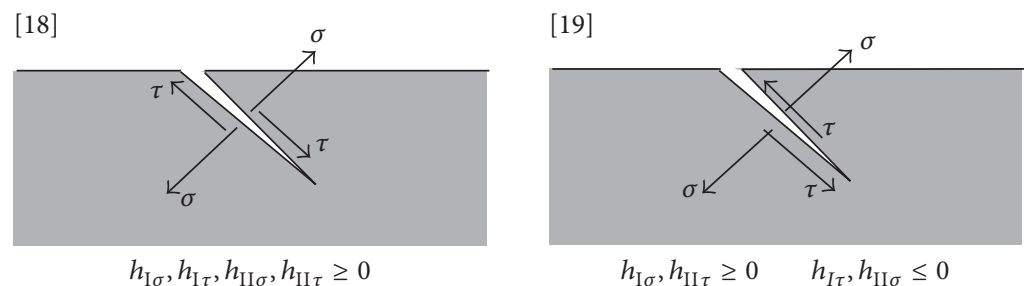

(b)

Figure 10: (a) Plots of the coefficients of the WFs in Eq. (A.1) from [18, 19]. (b) Conventional positive actions on the crack lips for the application of the WFs.

the conventions of the sign in $[18,19]$ are depicted for nominal stresses and SIFs. The signs of the WFs are also reported, which may help to eliminate the absolute values from functions in Figure 4.

In the literature, positive values of $K_{\mathrm{II}}$ are sometimes associated with nominal stresses shown in Figure 10(b) (left), as in [18], while in other cases they are associated with the opposite ones, Figure 10(b) (right), as in [19].

Given the polynomial expressions in (A.1), it is possible also to calculate the integrals in (25) in closed form

$$
\begin{aligned}
& K_{M}^{p}(d)=p_{m}(d) \int_{0}^{a} h_{M, \sigma}\left(x_{c}, a\right) d x_{c}=\sqrt{\frac{2}{\pi a}} p_{m}(d) \\
& \cdot \int_{0}^{a}\left[c_{0}^{M, \mu}\left(1-\frac{x_{c}}{a}\right)^{-0.5}\right. \\
& \left.+\sum_{i=1}^{4} c_{i}^{M, \mu}(\alpha)\left(1-\frac{x_{c}}{a}\right)^{i-0.5}\right] d x_{c} \\
& \quad=2 \sqrt{\frac{2 a}{\pi}} p_{m}(d)\left[c_{0}^{M, \mu}+\sum_{i=1}^{4} \frac{c_{i}^{M, \mu}(\alpha)}{2 i+1}\right]
\end{aligned}
$$


or

$$
\begin{gathered}
K_{M}^{p}(d)=p_{m}(d) \int_{0}^{a} h_{M, \sigma}\left(x_{c}, a\right)\left(1-\frac{x_{c}}{a}\right) d x_{c} \\
=\sqrt{\frac{2}{\pi a}} p_{m}(d) \int_{0}^{a}\left[c_{0}^{M, \mu}\left(1-\frac{x_{c}}{a}\right)^{-0.5}\right. \\
\left.+\sum_{i=1}^{4} c_{i}^{M, \mu}(\alpha)\left(1-\frac{x_{c}}{a}\right)^{i-0.5}\right]\left(1-\frac{x_{c}}{a}\right) d x_{c} \\
=2 \sqrt{\frac{2 a}{\pi}} p_{m}(d)\left[\frac{c_{0}^{M, \mu}}{3}+\sum_{i=1}^{4} \frac{c_{i}^{M, \mu}(\alpha)}{2 i+3}\right]
\end{gathered}
$$

\begin{tabular}{|c|c|}
\hline$a:$ & Crack length \\
\hline$a_{H}:$ & Hertzian contact half-width \\
\hline$d:$ & $\begin{array}{l}\text { Position of the travelling load; } \\
\text { the origin } \Omega \text { of } S_{t} \text { has } \\
\text { coordinates }(d, 0) \text { in } S\end{array}$ \\
\hline$f:$ & Coefficient of sliding friction \\
\hline$h_{\mathrm{I}}:$ & $\begin{array}{l}\text { Weight Function for } \\
\text { calculating } K_{\mathrm{I}}\end{array}$ \\
\hline$h_{\mathrm{II}}:$ & $\begin{array}{l}\text { Weight Function for } \\
\text { calculating } K_{\mathrm{II}}\end{array}$ \\
\hline$h_{\mathrm{I}, \sigma} / h_{\mathrm{I}, \tau}:$ & $\begin{array}{l}\text { Weight Function for } \\
\text { calculating the contribution } \\
\text { of } \sigma_{n} / \tau_{n} \text { to } K_{\mathrm{I}} \text { in oblique } \\
\text { cracks }\end{array}$ \\
\hline$h_{\mathrm{II}, \sigma} / h_{\mathrm{II}, \tau}:$ & $\begin{array}{l}\text { Weight Function for } \\
\text { calculating the contribution } \\
\text { of } \sigma_{n} / \tau_{n} \text { to } K_{\mathrm{II}} \text { in oblique } \\
\text { cracks }\end{array}$ \\
\hline$i$ : & Imaginary unit \\
\hline$p_{0}:$ & $\begin{array}{l}\text { Maximum Hertzian pressure } \\
\left(p_{0}>0\right)\end{array}$ \\
\hline$p_{m}:$ & Pressure at the crack mouth \\
\hline$z:$ & Complex variable \\
\hline A: & Airy's function \\
\hline$F_{\mathrm{I} / \mathrm{II}}=K_{\mathrm{I} / \mathrm{II}}\left(1 / p_{0} \sqrt{\pi a_{H}}\right):$ & Dimensionless SIFs \\
\hline$K_{\mathrm{I}}:$ & $\begin{array}{l}\text { Stress intensity factor for the } \\
\text { first mode (opening) }\end{array}$ \\
\hline$K_{\mathrm{II}}:$ & $\begin{array}{l}\text { Stress intensity factor for the } \\
\text { second mode (sliding) }\end{array}$ \\
\hline$K^{p}:$ & $\begin{array}{l}\text { Contribution of pressure } p \text { on } \\
\text { the crack lips to the stress } \\
\text { intensity factor (both for } \\
\text { modes I and II) }\end{array}$ \\
\hline$N:$ & $\begin{array}{l}\text { Normal actions along the } \\
\text { half-plane border ( }>0 \text { when } \\
\text { directed as the } y \text {-axis) }\end{array}$ \\
\hline R: & $\begin{array}{l}\text { Rotation matrix between } S_{c} \\
\text { and } S\end{array}$ \\
\hline
\end{tabular}

with $M=\mathrm{I}$, II, in order to easily take into account fluid pressure within the crack.

\section{Symbols}

$S\{O, x, y\}: \quad$ Fixed reference frame, $O$ at the crack mouth, $x$ along the border, and half-plane in $y \leq 0$

$S_{c}\left\{O, x_{c}, y_{c}\right\}:$ Fixed reference frame, $O$ at the crack mouth, $x_{c}$ along the crack faces towards the mouth

$S_{t}\left\{\Omega, x_{t}, y_{t}\right\}$ : Reference frame travelling with the load, axes parallel to S

T: $\quad$ Tangential actions along the half-plane boundary $(>0$ when directed as the $x$-axis)

T: $\quad$ Stress tensor $(2 \times 2)$

$\alpha: \quad$ Angle of the crack with respect to the surface

$\lambda$ : $\quad$ Corrective factor when the crack lips are closed, ratio between $\sigma_{n}$ and $\sigma_{c}$

$\sigma_{c}$ : Contact actions on the crack lips

$\sigma_{n}: \quad \quad$ Nominal normal stress along the crack lips in the uncracked body ( $>0$ when tractive; $0>$ when compressive)

$\tau_{n}: \quad$ Nominal shear stress along the crack lips in the uncracked body (arbitrary positive sign)

$\Phi / \Psi$ : $\quad$ Muskhelishvili’s potential functions.

\section{Conflicts of Interest}

The author declares that there are no conflicts of interest regarding the publication of this paper.

\section{References}

[1] T. Tallian, "Progress in rolling contact technology," Tech. Rep. Al690007, SKF Industries, 1969.

[2] H. A. Tedric, Rolling Bearings Analysis, John Wiley \& Sons, New York, NY, USA, 1991.

[3] D. A. Hills, A. Sackfield, and A. Uzel, "The green's function for a slant edge crack," Engineering Fracture Mechanics, vol. 20, no. 2, pp. 245-253, 1984.

[4] L. M. Keer and M. D. Bryant, "Pitting model for rolling contact fatigue," Journal of Lubrication Technology, vol. 105, no. 2, pp. 198-205, 1983.

[5] A. Nakajiama, K. Ichmaru, F. Hirano, and M. Nishimura, "Effects of combination of rolling direction and sliding direction on pitting of rollers," JSLE International, vol. 4, pp. 94-98, 1983.

[6] Y. Murakami and S. Nemat-Nasser, "Growth and stability of interacting surface flaws of arbitrary shape," Engineering Fracture Mechanics, vol. 17, no. 3, pp. 193-210, 1983.

[7] A. F. Bower, "Influence of crack face friction and trapped fluid on surface initiated rolling contact fatigue cracks," Journal of Tribology, vol. 110, no. 4, pp. 704-711, 1988. 
[8] O. P. Datsyshyn and H. P. Marchenko, "Stressed state of a half plane with shallow edge crack under Hertzian loading (a survey)," Journal of Materials Science, vol. 44, no. 1, pp. 22-34, 2008.

[9] M. Beghini and C. Santus, "An application of the weight function technique to inclined surface cracks under rolling contact fatigue, assessment and parametric analysis," Engineering Fracture Mechanics, vol. 98, no. 1, pp. 153-168, 2013.

[10] J. Seo, S. Kwon, H. Jun, and D. Lee, "Fatigue crack growth behavior of surface crack in rails," Procedia Engineering, vol. 21, no. 1, pp. 865-872, 2010.

[11] M. Dallago, M. Benedetti, S. Ancellotti, and V. Fontanari, "The role of lubricating fluid pressurization and entrapment on the path of inclined edge cracks originated under rolling-sliding contact fatigue: numerical analyses vs. experimental evidences," International Journal of Fatigue, vol. 92, pp. 517-530, 2016.

[12] T. L. Anderson, Fracture Mechanics: Fundamentals and Applications, CRC Press, 3rd edition, 2004.

[13] P. Kumar, Elements of Fracture Mechanics, Mc Graw Hill Education, 2017.

[14] S. Bogdański, M. Olzak, and J. Stupnicki, "Numerical stress analysis of rail rolling contact fatigue cracks," Wear, vol. 191, no. 1-2, pp. 14-24, 1996.

[15] S. Ancellotti, M. Benedetti, M. Dallago, and V. Fontanari, "The role of the second body on the pressurization and entrapment of oil in cracks produced under lubricated rolling-sliding contact fatigue," Theoretical and Applied Fracture Mechanics, vol. 91, pp. 3-16, 2017.

[16] R. Bassani, E. Ciulli, and F. Di Puccio, "Analysis of a surface crack in rolling bearings," in Proceedings of the NCBS '99 International Conference: Problems of Non-Conventional Bearing System, pp. 305-310, 1999.

[17] M. Beghini, L. Bertini, and V. Fontanari, "Parametric study of oblique edge cracks under cyclic contact loading," Fatigue \& Fracture of Engineering Materials \& Structures, vol. 28, no. 1-2, pp. 31-40, 2005.

[18] M. Beghini, L. Bertini, and V. Fontanari, "Weight function for an inclined edge crack in a semiplane," International Journal of Fracture, vol. 99, no. 4, pp. 281-292, 1999.

[19] T. Fett and G. Rizzi, "Weight functions for stress intensity factors and T-stress for oblique cracks in a half-space," International Journal of Fracture, vol. 132, no. 1, pp. L9-L16, 2005.

[20] T. Fett and D. Munz, "Stress intensity factors and weight functions," in Advances in Fracture Series, Computational Mechanics Publications, 1997.

[21] X. R. Wu and A. J. Carlsson, Weight Functions and Stress Intensity Factor Solutions, Pergamon Press, Oxford, UK, 1991.

[22] G. B. Airy, "On the strains in the interior of beams," Philosophical Transactions of the Royal Society A: Mathematical, Physical \& Engineering Sciences, vol. 153, pp. 49-79, 1863.

[23] N. I. Muskhelishvili, Some Basic Problems in the Theory of Elasticity, Noordhoff, Netherlands, 1953.

[24] D. Canadinc, H. Sehitoglu, and K. Verzal, "Analysis of surface crack growth under rolling contact fatigue," International Journal of Fatigue, vol. 30, no. 9, pp. 1678-1689, 2008.

[25] M. Kaneta and Y. Murakami, "Propagation of semi-elliptical surface cracks in lubricated rolling/sliding elliptical contacts," Journal of Tribology, vol. 113, no. 2, pp. 270-275, 1991. 


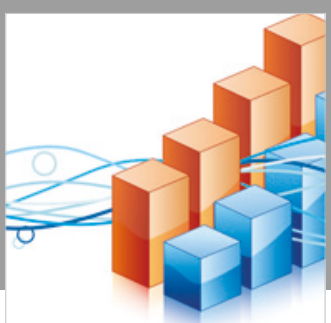

Advances in

Operations Research

\section{-n-m}
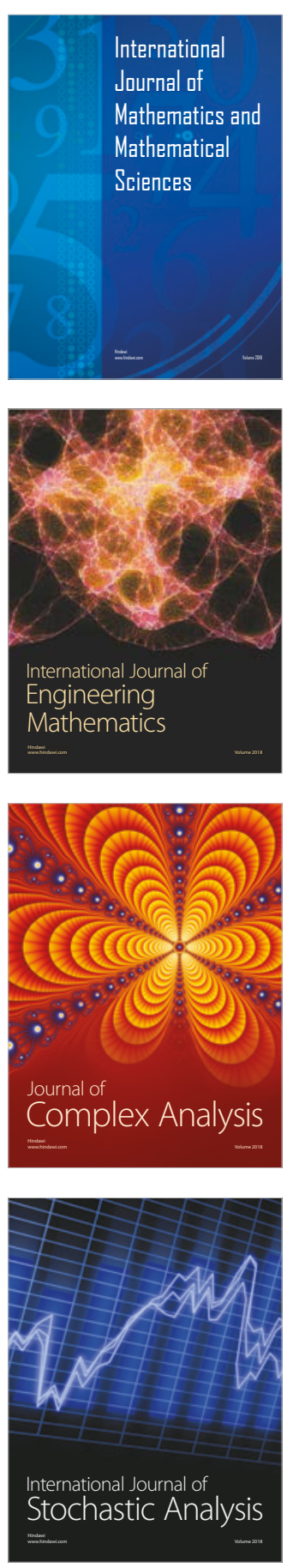
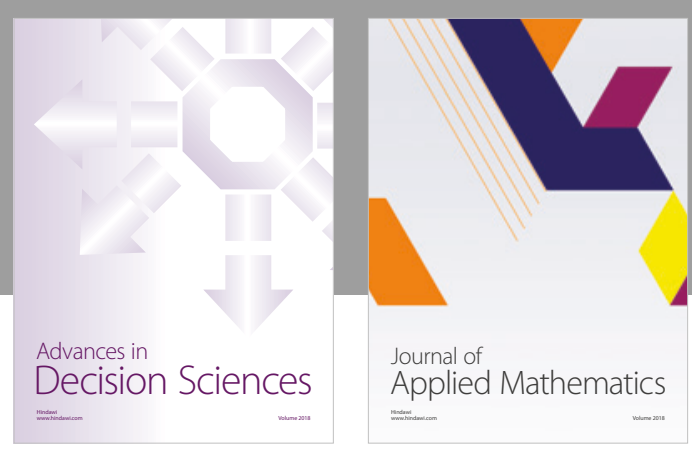

Journal of

Applied Mathematics
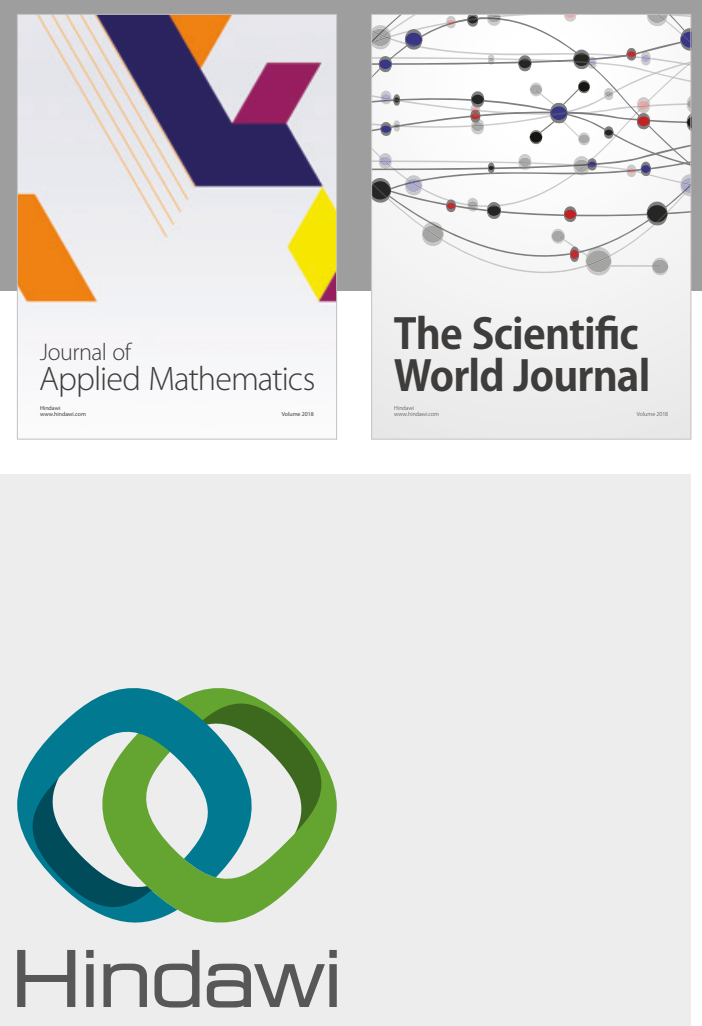

Submit your manuscripts at

www.hindawi.com

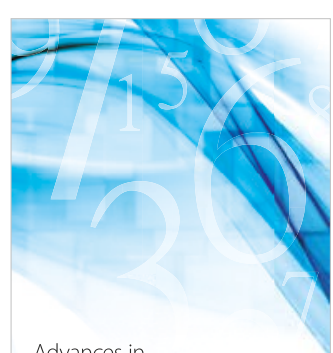

Advances in
Numerical Analysis
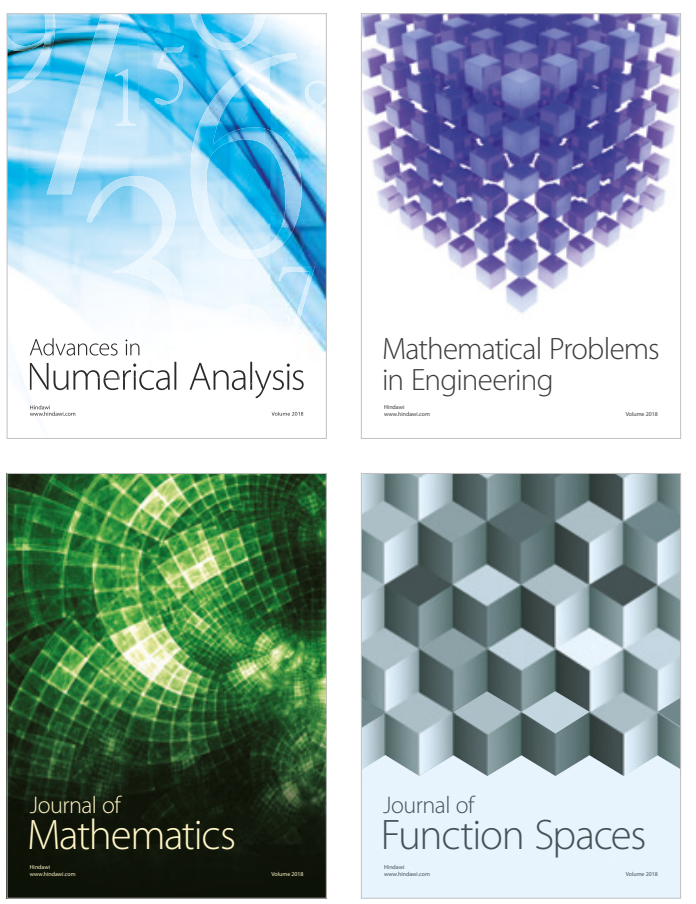

Mathematical Problems in Engineering

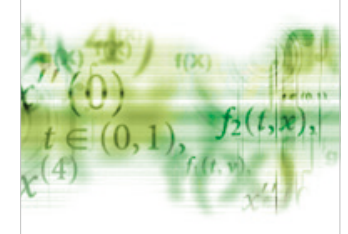

International Journal of

Differential Equations

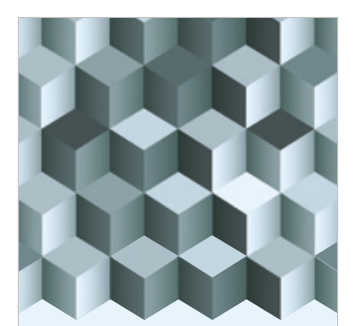

Journal of

Function Spaces

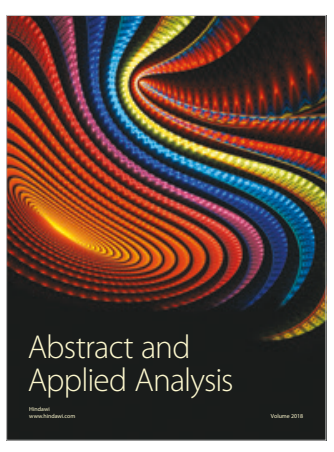

The Scientific

World Journal

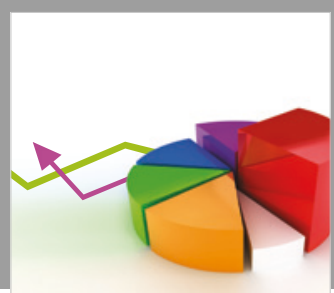

Journal of

Probability and Statistics
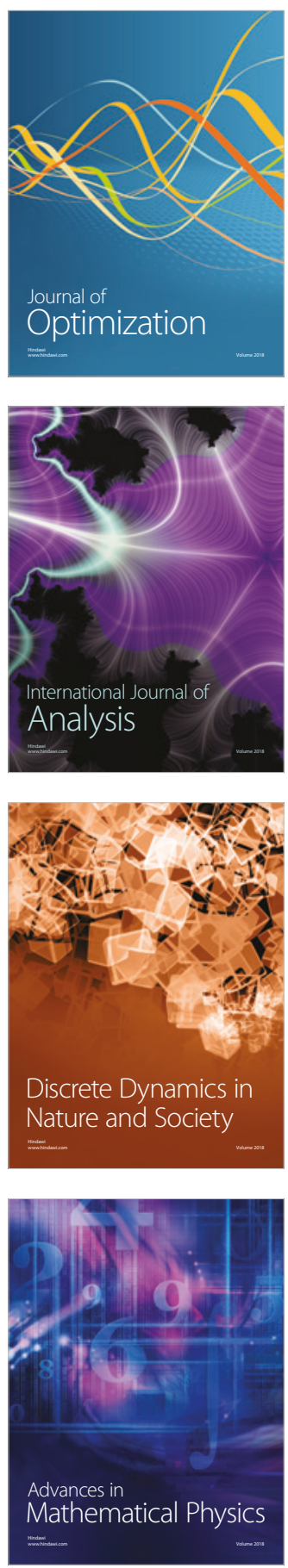